\title{
LISTE FLORISTIQUE DE LA VEGETATION PERENNE DU JARDIN GUERRA JUNQUEIRO (LISBONNE) EN 1995
}

(PERENNIAL VEGETATION FLORISTIC LIST OF GUERRA JUNQUEIRO GARDEN - LISBON - IN 1995)

\section{EUGÉNIA S. Albergariâ MOREIRA ${ }^{1}$}

La composition floristique de la végétation perenne du Jardin Guerra Junqueiro (ou J. da Estrela), entre Janvier 1994 et le 10 Décembre 1995, quand le dernier prelèvement floristique a été fini, présente 306 taxa, parmi espèces (sp.), sous-espèces (ssp.), varietés (var.), formes (for.), hybrides et cultivares. Ces taxa sont integrés en 196 genres et 94 familles, selon la nomenclature taxonomique presentée par HUXLEY et all. (1992) et GRIFFITS (1994). La liste est organisée selon l'ordre alphabétique des familles.

La densité floristique de 70,12 taxa/ha (44,91genres/ha et 21,54 familles/ha), est assez élevée pour un petit jardin de 4,364 ha. Mais ces valeurs sont en accord avec les caractéristiques architectoniques du jardin, d'origine romantique et avec une longue tradition de collection botanique, aussi bien qu'avec les caractéristiques d'un milieu naturel tipique de l'écotone atlantique-meditérranéen-sous-tropical.

\section{LISTE FLORISTIQUE \\ (FLORISTIQUE LIST) \\ (le 10 Décembre, 1995)}

ACANTHACEAE Juss.

Acanthus L.

A. mollis L.

Justicia L.

J. adhatoda L.

J. carnea Lindl.

ACERACEAE Juss.

Acer L.

A. negundo L.
AGAVACEAE Endl.

Agave L.

A. americana $\mathrm{L}$.

A. americana L. "Marginata" Trel.

A. ferox Koch.

Cordyline Comm. Ex R. Br.

C. australis (Forst.) Endl.

Dracaena Vand. Ex L.

D. draco L.

${ }^{1}$ Investigadora do Centro de Estudos Geográficos da Universidade de Lisboa. Professora Catedrática da Universidade de Lisboa. (Endereço do CEG no início do volume). 
Furcraea Vent.

$F$. foetida (L.) Haw.

Nolina Michx.

N. recurvata (Lem.) Hemsl.

Phormium Forst. \& Forst. f.

$P$. tenax Forst. \& Forst. f.

P. tenax Forst. \& Forst. f. "Marginatum" Yucca L.

Y. gloriosa L.

Y. treculeana Carrière

AMARANTHACEAE Juss.

Iresine P. Browne

I. herbstii Hook

I. lindenii Van Houte

AMARYLLIDACEAE J. St.-Hil.

Clivia Lindl.

C. $x$ cyrtanthiflora (Van Houte) Wittm.

Crinum L.

C. miniata Reg.

C. moorei Hook.

ANACARDIACEAE Lindl.

Harpephyllum Bernh. ex K Krause

H. caffrum Bernh. ex K. Krause Schinus L.

S. molle L.

S. terebinthifolius Raddi

APOCYNACEAE Juss.

Nerium L.

$N$. oleander L.

N. oleander L. "Albaplenum"

$N$. oleander L. "Luteum"

Vinca L.

$N$. oleander L. "Rosaplenum"

V. difformis Pourr.

ARACEAE Júss.

Arum L.

A. italicum Mill.

Monstera Adans.

M. deliciosa Liebm.

Zantedeschia Spreng.

Z. aethyopica (L.) Spreng.
ARALIACEAE Juss.

$x$ Fatshereda Guill.

x F. lizei Guill.

Fatsia Decne \& Planch.

F. japonica (Tunb.) Decne \& Planch.

Hedera L.

$H$. canariensis Willd.

H. canariensis Willd. "Arborescens"

H. helix L. "Gloire de Marengo"

Schefflera Forst. \& Forts. f.

S. pubigera (Brongn. ex Planch.) Frodin

ARAUCARIACEAE Henkel \& Hochst.

Araucaria Juss.

A. columnaris (Forst. f.) Hook.

A. heterophylla (Salisb.) Franco

ARECACEAE (PALMAE) C. H. Schultz Butia Becc.

B. capitata (Mart.) Becc.

Chamaedorea Willd.

C.elegans Mart.

Chamaerops L.

C. humilis L. var. arborescens (Pers.)

Steud.

Howea Becc.

H. belmoreana (C. More \& F. Mueller)

Becc.

H. forsteriana (C. More \& F. Mueller) Becc.

PhoenixL.

$P$. canariensis hort. ex Chabaud

$P$. reclinata Jacq.

Rhopalostylis H. A. Wendl. \& Drude

$R$. baueri (Seem.) H. A. Wendl. \& Drude Syagrus Mart.

S. romanzoffiana (Cham.) Glassman

Washingtonia $\mathrm{H}$. A. Wendl.

W. robusta $\mathrm{H}$. A. Wendl.

ARISTOLOCHIACEAE JusS.

Asarum L.

A. canadense $\mathrm{L}$.

BALSAMINACEAE A. Rich.

Impatiens L.

I. sodenii Engl. \& Warb. ex Engl.

I. walleriana Hook. f. 
BERBERIDACEAE JusS.

Berberis L.
B. gagnepainii Schneid.
B. juliana Schneid.
B. $x$ stenophylla Lindley

\section{BETULACEAE Gray}

Betula L.

B. celtiberica Rothm. \& Vasc.

BIGNONIACEAE Juss.

Jacaranda Juss.

J. mimosifolia D. Don.

BLECHNACEAE (C. Presl.) Copel

Woodwardia Sm.

$W$. radicans (L.) Sm.

BOMBACACEAE Kunth.

Chorisia kunth.

C. speciosa A. St.-Hil.

BORAGINACEAE Juss.

Echium L.

E. candicans L.f.

Trachystemon D.Don

T. orientalis (L.) G.Don

\section{BROMELIACEAE Juss.}

Bilbergia Thunb.

B. vittata Brongn. ex Morel

BUXACEAE Dumort.

Buxus L.

B. sempervirens L.

CACTACEAE Juss.

Cereus Mill.

C. uruguaianus Kiesling

C. uruguaianus Kiesling "Monstruosus"

CANNACEAE Juss.

Canna L.

C. $x$ generalis L. H. Bailey "America"

C. $x$ generalis L. H. Bailey "Cleopatra"

C. $x$ generalis L. H. Bailey "Lucifer"

C. $x$ generalis L. H. Bailey "Rigoleto"
CAPRIFOLIACEAE Juss.

Abelia R. Br.

$$
\text { A. } x \text { grandiflora (André) Rehder }
$$

Lonicera L.

L. japonica Thunb.

Sambucus L.

S. nigra L.

Viburnum L.

V. opulus L.

V. suspensum Lindl.

V. tinus L. ssp. tinus

V. tinus L. ssp. tinus "Variegatum"

Weigela Thunb.

W. florida (Bunge) A. DC.

CASUARINACEAE R. Br.

Casuarina Adans.

C. cunninghamiana Miq.

\section{CELASTRACEAE}

Euonymus L.

E. japonicus Thunb.

E. japonica Thunb. "Albomarginatus"

E. japonica Thunb. "Aureomarginatus"

E. japonicus Thunb. "Aureus"

E. japonica Thunb. "Medio Pictus"

COMMELINACEAE R. Br.

Tradescancia $\mathrm{L}$.

T. fluminensis Vell.

COMPOSITAE Giseke

Ageratina Spach.

A. adenophora (Spreng.) R.King \& H.

Robinson

Ageratum L.

A. houstonianum Mill

Arctotis L.

A. acaulis $\mathrm{L}$.

Argyranthemum Webb ex Schultz-Bip.

A. frutescens $\mathrm{L}$.

Leucanthemum Mill.

L.vulgare Lam.

Montanoa La Ll. \& Lex.

M. bipinnatifida (Kunth) K. Koch 
Roldana La Ll. \& Lex.

$R$. petasites (Sims) H. Robinson \& Brettel Senecio $L$.

S. cineraria $\mathrm{DC}$.

Tanaceutum $\mathrm{L}$.

T. parthenium (L.) Schultz-Bip.

CORNACEAE Dumort. (AUCUBACEAE J. Agardh.)

Aucuba Thunb.

A. japonica Thunb. "Variegata"

CORYNOCARPACEAE Engl.

Corynocarpus Forst. \& Forst. f.

C. laevigata Forst. \& Forst $\mathrm{f}$.

CRASSULACEAE DC.

Crassula L.

C. ovata (Mill.) Druce

Kalanchoe Adans.

$K$. daigremontiana Hamer \& Perrier

CUPRESSACEAE Bartling

Cupressus L.

C.sempervirens $\mathrm{L}$. for.sempervirens Juniperus L.

J. turbinata (Guss.) Nyman

Sequoia Endl. Thuja L.

$S$. sempervirens (D. Don) Endl

T. occidentalis $\mathrm{L}$.

T. plicata D. Don.

CYATHEACEAE Kaulf.

Cyathea J. Sm.

C. cooperi (F.Mueller) Domin

CYCADACEAE Pers.

Cycas L.

C. revoluta Thunb.

\section{CYPERACEAE Juss.}

Carex L.

Cyperus L.

C. pendula Huds.

C. involucratus Rottb.
DRYOPTERIDACEAE Ching.

Cyrtomium Presl.

C. falcatum (L.f.) Presl.

Polystichum Roth.

$P$. setiferum (Forssk.) Woyn.

ELAEAGNACEAE Juss.

Elaeagnus L.

E. angustifolia $\mathrm{L}$.

ERICACEAE Juss.

Arbutus (Tourn.) L.

A. unedo $\mathrm{L}$.

EUPHORBIACEAE Juss.

Euphorbia L.

E. pulcherrima Willd. ex Klotzsch.

FAGACEAE Dumort.

Quercus L.

Q. ilex ssp. rotundifolia (Lam.) Tab.

Morais

Q. robur $\mathrm{L}$.

FLACOURTIACEAE DC.

Dovyalis E. Mey.

D. caffra Warb.

GERANIACEAE Juss.

Pelargonium L'Hérit

$P$. $x$ domesticum L. H. Bailey

P. $x$ hortorum L. H. Bailey

GINKGOACEA Engl.

Ginkgo L.

G. biloba L.

GROSSULARIACEAE DC.

Escallonia Mutis ex L. f.

E. rubra (Ruiz \& Pav.) Pers. var.

macrantha (Hook \& Harn) Reiche.

GUTTIFERAE Juss.

Hypericum L.

H. calycinum $\mathrm{L}$.

HIPPOCASTANACEAE DC.

Aesculus L.

A. $x$ carnea Hayne

A. hippocastanum $\mathrm{L}$. 
HYDRANGEACEAE Dumort.

Deutzia Thunb.

D. $x$ magnifica (Lemoine) Rehd.

Hydrangea L.

H. macrophylla (Thunb.) Ser. subsp. macrophylla

Philadelphus L.

$P$. coronarius $\mathrm{L}$.

IRIDACEAE Juss.

Iris L.

I. germanica $\mathrm{L}$.

I. germanica L. "Florentina"

I. unguicularis Poir.

JUNCACEAE Juss.

Juncus L.

$J$. inflexus L.

\section{LABIATAE Juss. (LAMIACEAE)}

Lamium L.

L. galaeobdolon (L.) L. "Variegatum" Plectranthus L'Hérit

$P$. forsteri Benth.

Salvia L

$P$. fruticosus L'Hérit

S. microphylla Kunth.

S. splendens Sellow ex Roem. \& Schult.

"Grandiflora"

S. splendens Sellow ex Roem. \& Schult. "Nana"

\section{LARDIZABALACEAE Decne}

Akebia Decne

A. quinata (Hout.) Decne

LAURACEAE Juss.

Apollonias

A. barbusana (Cav.) Bornm.

Laurus L.

$L$ nobilis $\mathrm{L}$.

Ocotea

O. foetens (Ait.) Benth. \& Hook f.

LEGUMINOSAE Juss.

CAESALPINIOIDEAE

Bauhinia L.

B. forficata Link.
Ceratonia L.

C. siliqua $\mathrm{L}$.

Gleditsia L.

G. triacanthos $\mathrm{L}$.

MIMOSOIDEAE

Acacia Mill.)

A. Karoo Hayne

A. melanoxylon $R$. Br.

PAPILIONOIDEAE

Cercis L.

C. siliquastrum $\mathrm{L}$.

Erythrina L.

E. caffra Thunb

E. coralloides DC.

E. crista-galli $\mathrm{L}$.

Robinea L.

$R$. pseudoacacia L.

Sophora L.

$S$. japonica L

Tipuana Benth.

T. tipu (Benth.) O. Kuntze

LILIACEAE Juss.

Agapanthus L'Hérit

A. praecox Willd. subsp. orientalis

(F. M. Leighton) F. M. Leigthon

A. praecox Willd. subsp. orientalis

(F. M. Leighton) F. M.Leigthon "Albus" Aloe L.

A. arborescens Miller

A. ciliaris Haw.

A. saponaria (Ait. f.) Haw.

Asparagus L.

A. densiflorus (Kunth) Jessop "Sprengeri"

A. falcatus $\mathrm{L}$.

A. setaceus (Kunth) Jessop

Chlorophytum Ker-Gawl.

C. comosum (Thunb.) Jacques

C. comosum (Thunb.) Jacques

"Variegatum"

Hemerocallis L.

$H$. fulva $\mathrm{L}$.

Rohdea Roth.

$R$. japonica (Thunb.) Roth "Marginata"

Ophiopogon Ker-Gawl.

O. jaburan (Kunth) Lodd.

O. japonicus (L. f.) Ker-Gawl. 
Ruscus L.

R. aculeatus $\mathrm{L}$.

R. hypophyllum $\mathrm{L}$.

LOGANIACEAE C. Mart.

Buddleja L.

B. davidii Franch. var. magnifica Rehed.

\& Wils.

\section{LYTHRACEAE St.-Hil.}

Cuphea P. Browne

C. micropetala $\mathrm{HBK}$

Heimia Link.

H. salicifolia (HBK) Link.

Lagestroemia L.

L. indica $\mathrm{L}$.

MAGNOLIACEAE Juss.

Magnolia L.

M.grandiflora L.

MALVACEAE Juss.

Hibiscus L.

H. rosa-sinensis $\mathrm{L}$.

H. rosa-sinensis L. "Cabo"

H. syriacus $\mathrm{L}$.

H. syriacus L. "Blue Bird"

H. syriacus L. "Monstrosus"

H. syriacus L. "Puniceus Plenus"

Lagunaria G. Don.

L. patersonii (Anderss.) G. Don.

Malvaviscus Adans.

M. arboreus Cav.

Phymosia Desv.

P. umbelata (Cav.) Kearn

MORACEAE Link.

Ficus L.

F. altissima $\mathrm{Bl}$.

F. drupaceae Thunb.

Ficus elastica Roxb. ex Hornem

Ficus elastica Roxb. ex Hornem

"Decora"

F. macrophylla Desf. ex Pers.

F. rubiginosa Desf. ex Vent.

F. watkinsiana Bail.
MYOPORACEAE R. Br.

Myoporum Banks\& Sol. ex Forst. f.

M. tenuinfolium Forst. f.

MYRTACEAE JuSs.

Acca O. Berg.

A. sellowiana (O. Berg) Burret

Callistemon R. Br.

C. speciosus (Sims.) DC.

Eugenia L.

E. uniflora $\mathrm{L}$.

Metrosideros Banks ex Gaertn.

$M$. excelsa Sol. ex Gaertn.

Myrthus L.

M. communis L.ssp. communis $\mathrm{L}$.

Rhododendron $\mathrm{L}$.

R. mucrunatum G. Don.

NYCTAGINACEAE Juss.

Bougainvillea Comm. ex Juss.

B. glabra Choisy in DC.

Mirabilis L.

M. jalapa $\mathrm{L}$.

OLEACEAE Hoffsgg. \& Link.

Fraxinus L.

$F$. angustifolia Vahl.

Jasminum L.

J. fruticans $\mathrm{L}$.

$J$. mesnyi Hence

Ligustrum L.

L. indicum (Lour.) Merrill.

L. japonicum Thunb.

L. lucidum Ait.

L. lucidum Ait. "Aureovariegatum"

L. ovalifolium Hassk.

Phillyrea L.

Olea L.

P. latifolia $\mathrm{L}$.

O. europaea L. var. europaea L.

O. europaea L. var. oleaster (Hoffm. \& Link.) DC.

Syringa $\mathrm{L}$.

S. vulgaris L.

S. vulgaris L."Mme. Lemoine" 
Pyracantha Roem.

P. angustifolia (Franch.) Schneid.

$P$. coccinea Roem.

P. crenatoserrata (Hance) Rehd.

Rhaphiolepis Lindl.

R. x delacourii André

Rosa $L$.

R. canina $\mathrm{L}$

R. chinensis Jacq. "Cécile Brunner Repens"

R. chinensis Jacq. "Minima"

Rosa sps.

Spiraea L.

S. cantoniensis Lour.

S. trilobata L.

RUBIACEAE Juss.

Coprosma Forst.

C. repens A. Rich. "Marginata"

SALICACEAE Mirb.

Populus L.

P. alba L. "Pyramidalis"

$P$. nigra L.

P. nigra L. "Italica"

Salix L.

S. babylonica L.

SAXIFRAGACEAE Juss.

Bergenia Moench.

$B$. crassifolia (L.) Fritsch.

SCROPHULARIACEAE JuSS.

Freylinia Colla.

F. lanceolata (L.f.) G. Don

Hebe Comm. ex Juss.

H. $x$ andersonii (Lindl. \& Paxton)

Cokayne

H. salicifolia (Forts. f.) Pennell.

H. speciosa (A.Cunn.) Cukaine \& Allan.

SIMAROUBACEAE DC.

Ailanthus Desf.

A. altissima (Mill.) Swingle
SOLANACEAE Juss.

Brugmansia Pers.

B. arborea (L.) Lagehrt

B. $x$ candida Pers.

B. sanguinea (Ruiz \& Pav.) D.Don

Cestrum L.

C. aurantiacum Lindl.

C. $x$ cultum Francey

C. fasciculatum (Schldl.) Miers

C. roseum $\mathrm{HBK}$.

Iochroma Benth.

I. coccinea Scheidw.

I. cyanea (Lindl.) Green.

Solanum L.

S. aviculare Forst. f.

S. pseudocapsicum L.

$S$. umbelliferum Eschsch.

STERCULEACEAE Bartad

Brachychiton Schott \& Endl.

B. populneus (Schott \& Endl.) R. Br. Dombeya Cav.

D. $x$ cayeuxii André

STRELITZIACEAE (Schumann) Hutch.

Strelitzia Banks ex Dryand.

S. nicolai Regel \& Körn

$S$. reginae Banks ex Dryand.

TAMARICACEAE Link.

Tamarix $\mathrm{L}$.

T. parviflora DC.

THEACEAE D. Don.

Camellia L.

C. japonica L. "Alba Plena"

C. japonica L. "Anticipation"

C. japonica L. "Elegans"

C. japonica L. "Lovelight"

C. japonica L. "Magnolifflora"

C. japonica L. "Miss Charleston Var."

C. japonica L. "Nobilissima"

C. japonica L. "Tomorrow"

C. sasanqua Thunb.

TILIACEAE Juss.

Sparmannia L.f.

S. africana L.f. 
Tilia L.

T. platyphyllos Scop.

T. tomentosa Moench

T. $x$ vulgaris Hayne

ULMACEAE Mirb.

Celtis L.

C. australis $\mathrm{L}$.

Ulmus L.

U. carpinifolia Rupp. Ex Suckow

$U$. glabra Huds.

URTICACEAE Juss.

Soleirola Gaudich.

S. soleirolii (Req.) Dandy

VALERIANACEAE Batsch.

Centranthus Necker ex DC.

C. ruber (L.) DC.
VERBENACEAE St.-Hil.

Duranta L.

D. erecta L.

Lantana L.

L. camara $\mathrm{L}$.

Vitex $\mathrm{L}$.

V. agnus-castus $\mathrm{L}$

VITACEAE Juss.

Parthenocissus Planch.

P. quinquefolia (L.) Planch.

ZINGIBERACEAE Lindl.

Alpinia Roxb.

A. malaccensis (Burm.) Roscoe

Hedychium J. G. König

H. gardnerianum Ker-Gawl.

\section{NOTE FINALE}

Ce travail est un résultat du Project de Recherche JNICT-PCS/C/GEO/712/93 du Project Estímulo para as Ciências Sociais e Humanas " Espaços verdes de Lisboa, Ambiente, Imagem e Utilização". Nous remercioms Mme. le Docteur Cândida Liberato, de l'I.I.C.T. de Lisbonne et M. le Professeur Mário Lousã, de l'I.S.A. - Département de Botanique, qui ont bien voulu identifier les espèces de Ficus.

\section{BIBLIOGRAPHIE}

BONELLS, J. E. (1983) - Plantas y Jardines de Sevilla. Sevilha, Ayuntamiento de Sevilla, Delegación de Parques y Jardines, 340 p.

BOWN, D. (1995) - Encyclopedia of Herbs and Their Uses. $1^{\mathrm{a}}$ edição. The Royal Horticultural Society. Londres, Dorling Kindersley, $424 \mathrm{p}$.

BRICKELL, Ch., ZUK E. (Edt.) (1997) - A-Z Encyclopedia of Garden Plants. The American Horticultural Society, New York, DK Publishing, Inc.

CAIXINHAS, M. L. (1994) - Flora da Estufa Fria de Lisboa. Lisboa, Edit. Verbo, 143 p. 143.

CHOUKAS-BRADLEY, M.; ALEXANDER P. (1987) - City of Trees. The Complete Field Guide to the Trees of Washington, D.C. Baltimore, The Johns Hopkins University Press, $354 \mathrm{p}$.

COOMBES, A. (1994) - Dictionary of Plant Names. Portland (Oregon), Timber Press, 195 p.

COURTRIGHT, G. (1995) - Tropicals. Portland (Oregon), Timber Press, 155 p.

GONZALEZ, G, L. (1982) - La Guia de Incafo de los Árboles y Arbustos de la Península Iberica. Madrid, Incafo, $866 \mathrm{p}$.

GRAF, A. B. (1981) - Tropica, Color Cyclopedia of Exotic Plants and Trees. East Rutherford (New Jersey), Rohers Company. 
(1992) - Hortica. Color Cyclopedia of Flora and Indoor Plants. East Rutherford (New Jersey), Rohers Company.

GRIFFITS, M. (1994) - Index of Garden Plants. The New Royal Horticultural Society Dictionary. Londres, Macmillan Publishers, Ltd.

HOLLIDAY, I.; HILL, R. (1987) - A Field Guide to Australian Trees. Chatswood (N.S.W.), Rigby Publishers, $230 \mathrm{p}$.

HOLLIDAY I,; WATTON, G. (1987) - A Field Guide to Australian Shrubs. Chatswood (N.S.W.), Rigby Publishers, 219 p.

HORTUS THIRD(1976).-. A Concise Dictionary of Plants Cultivated in the United States and Canada N. York, Liberty Hyde Baily Publis.

HUXLEY, A.; GRIFFITS, M.; LEVY, M., Edit. (1992) - Dicitionary of Gardening. The Royal Horticultural Society. Volumes I (A-C, 810 p.), Volume II (D-K, 747p.), Volume III (L-Q,747p.), Volume IV (R-Z, 888p.). Londres Macmillan Publishers, Ltd.

LIBERATO, M. C.; CHICHORRO M. A. (1994) - Catálogo de Plantas do Jardim-Museu Agrícola Tropical. Lisboa, Instituto de Investigação Científica Tropical e Fundação Berardo.

LILLO, A. L.; MIELGO M. (1995) - Árboles de Madrid. Madrid, Comunidad de Madrid, Consejeria de Cooperación, Agencia de Medio Ambiente, $4^{\mathrm{a}}$ edição, 212 p.

LORENZI, H. (1992) - Árvores Brasileiras. Manual de Identificação e Cultivo de Plantas Arbóreas Nativas do Brasil. Nova Odessa, SP., Ed. Plantarum, 352 p.

MABBERLY, D. D. (1993) - A portable dictionary of the higger plants. Cambridge University Press, $707 \mathrm{p}$.

MCHOY, P. (1997) - The American Pratical Garden Encyclopedia. N. York, Lorenz Books, $256 \mathrm{p}$.

ROACH, M. (1993) - Groundcovers. American Gardeningg Series. New York, Prentice Hall Gardening, $96 \mathrm{p}$.

SOARES, C. B. da V. (1990) - Árvores Nativas do Brasil. Rio de Janeiro (R.J.), Salamandra Consultoria Editorial S. A., 115 p.

STAROSTA, P. Ed. (1997) - Roses. Paris, Hachette Livre.

TESTU, Ch. (1970) - Conifêres de nos jardins. Paris, la Maison Rustique.

TESTU. Ch (1973) - Abres et Arbrisseaux de nos jardins. Paris, La Maison Rustique.

THE EUROPEAN GARDEN FLORA, Vol I - Pteridophytae, Gimnospermae, Angiospermae;

Alismataceae to Iridaceae - (1990). Londres, Cambridge University Press.

TORRE, J. R. de la et all. (1982) - Aproximación al catálogo de plantas vasculares de Madrid. Madrid, Comunidad de Madrid, Consejeria de Agricultura.

ZUCKER, I.; FELL, D. (1995) - Flowering Shrubs and Small Trees. N. York, Friedmann/ Fairfax Publishers. 